\title{
On the Disordered, Collective and Patterned Movements of Media. Qualitative Analysis of Movements
}

\author{
Géza Lámer ${ }^{\star}$ \\ 1 Department of Engineering Management and Enterprise, \\ Faculty of Engineering, University of Debrecen, \\ H-4028 Debrecen, Ótemető u. 2-4. \\ * Corresponding author, e-mail: glamer@eng.unideb.hu
}

Received: 04 January 2019, Accepted: 19 May 2019, Published online: 12 June 2019

\begin{abstract}
The motion of elements is classified from a topological aspect. We separate three different, disordered, collective and patterned motion forms of media. We will reveal what concrete shapes the different type media take. In case of a limited number of particles, a discrete description is suitable for tracking the mechanic behavior of the medium. In case of the large of a number of particles we apply in some sense continuous mathematical model according to the motion occurring in the medium instead of a discrete description. We postulate a statistical distribution to the disordered motion; we "distribute" the particles for the collective motion in the physical space and apply a differential geometric description. We assign continuous flow lines to the regular patterned motion of the medium with free particles while we assign energy dissipation to the flow image an irregular patterned motion. In the case of deformable solid body built by periodically arranged rigid bodies the state functions with discrete domain of definition are represented by functions with continuous domain of definition. Granular conglomerations seem to be the only such medium, which allow tracking of the state of all granules.

Disordered motion leads to thermodynamic - statistical description. The collective motion of free particles leads to the description of the laminar flow of particles, the collective motion of fixed particles leads to the classical continuum. The patterned motion of free particles leads to the description of vortex or turbulent flow, the patterned motion of fixed particles leads to grid continua.
\end{abstract}

\section{Keywords}

disordered, collective and patterned motion, discrete and continuous mechanical models

\section{Introduction}

Due to the high number of elements (more than $10^{23}$ ) constituting the medium, we cannot describe the mechanical behavior of medium by tracking of the independent motion of its each and every element. Therefore, instead of the individual analysis of each and every elements, we examine the domain occupied by the medium in the Euclidean space. Although, in the Euclidean space, we extend our examinations to a continuum number of many "particles" (points constituting the Euclidean space) during the examination of its domain, but by using the characteristics of continuity fundamentally as many equations have to be set up for domain as in the case of one particle. The difference is the following. In the case of equilibrium, instead of system of algebraic equation a boundary value problem for partial differential equations according to the space coordinates, and in the case of motion, instead of initial value problem for a system of ordinary differential equation an initial and boundary value problem for a system of partial differential equations according to the space and time coordinates have to be set up and solved.

Thus, formally the "task" is to switch to functions with continuous domain of definitions from functions with discrete domain of definition [1]. It is obvious, based on previous research, that either we handle the arising problem as a numerical method [2-4], or we take a geometric view instead of a discrete locus and we reinterpret the Newtonian mechanics on a continuum (see e.g. [1]). The latter method, which primarily applies the tools of differential geometry $[5,6]$, can be applied not only to elasticity, see e.g. [7, 8], but to the flow of liquids, see e.g. [9, 10], and also generally to the continuous description of the physical behavior of media [11]. 
The physical behavior of solid matter is diversified (see e.g. [12]). The continual approach in case of different "continuous" material behavior makes various description through material constants $[13,14]$ possible. However continuous description mode allows the modelling of some non-continuous behavior by means of discontinuity surfaces. The principle is applied primarily to the flow of liquids/gases (see e.g. $[9,10,15]$ ), but the mechanical behavior of stone mass "weakened" by discontinuities caused by fractures [16], or the "average" behavior of the structurally changing (degrading, crushing) grain set can be modelled by continuous description method [17] too. Continuous modeling provides discussion different material behaviors (elastic, thermal expansion, plastic and rheological processes) through a unified view of the metric tensor [18]. At the same time we believe that many material behaviors can be traced back to a few simple "general" motion forms. Therefore we seek those steps of modelling, where these simple "general" motion forms can still be detected.

The aim of the modelling is to forecast the phenomenon. The numerical method from this aspect plays a significant role in the theory. However, it is known from astronomy and celestial mechanics that although a numerical method in itself (the orbit of planet consist of movements on a few circles; the planet moves on a circle, the center of the circle moves on another circle, and so on) can provide an arbitrarily accurate forecast for some kinematic questions, but this will not reveal the cause of the motion. In fact, it will not even visibly reflect the nature of the trajectory of the actually occurring motion (see e.g. [19], or [20]). Keeping this in mind, we believe that in case of switching from a set of discrete particles (points) to a domain of the continuum, the characteristics of the motion of discrete particles have to be considered and such description has to be chosen which is not in contradiction with the nature of the motion of particles. This latter view determines the scope of this study and the order of the examinations:

- characteristics of particle motions within a body,

- models of bodies based on the motion of particles constituting the body,

- possibilities of describing disordered, collective and patterned motions.

The material is interpreted as a combination of corpuscles and interactions between them [21]. Material point, rigid and deformable solid body models are well suited for modeling corpuscles. The force fields between the bodies, in classical physics, can be well approached as force by Newton's $3^{\text {rd }}$ Law [21]. This approach is used in the study.
When examining the state of the material, the movements of the particles within the material, the existence of the atom-electron internal structure is not taken into account, and the atoms are "imagined" as rigid (or may be deformable) balls. However, the question of what the atoms hold together in liquid or solid state, and how the atoms "behave" in gas during collisions, is not explored.

\section{Description of particle motions within a body}

\subsection{Separating bodies based on state of matter}

\subsubsection{Separation of states based on the stability of shape} and volume, and the ratio of bond to kinetic energy

A material made up of physically and chemically identical particles is called a body. Bodies are classified into three groups based on their states of matter: gaseous, liquid and solid (see e.g. [21] or [22]). In order to distinguish between the three states, the classification is primarily based on the aspect of the shape/volume conservation/ change; see the first two columns of Table 1.

Classification of states can be done on the basis of the ratio of the bond and the kinetic energy dependent on the heat movement (see e.g. [21]), the two classifications lead to essentially the same result; see the last column of Table 1. The classification is complete, i.e., no further state can be separated according to these two aspects. Regardless of this, in physics, the fourth (plasma; see e.g. [21] or [22]) is sometimes distinguished. Plasma status is separated from the gaseous state by the appearance of electrically charged particles (see, for example, [21] or [22]).

Liquid, gaseous, and plasma states are commonly referred to as liquid states. In this grouping, the separation from the solids is based on the fact that, in the case of the continuous effect of a shear "force" in the liquid state, the body changes its shape continuously (see e.g. [22], [23] and [10]). Although page [22] also includes the plastic flow state solid bodies, the latter is in principle another material behavior: the plastic flow occurs only if the plastic flow condition exists (see e.g. [24] or [25]), until fluid flow is only triggered by shear "force".

\subsubsection{Separation of states based on the contact and the movement of the particles forming the body}

In the three different states of body, the particles that form the body come into contact with each other, as well as move in different ways. Contact and movement can be considered as a possible classification criterion (see for example [26]). The three states can have different characteristics; see Table 2. 
Table 1 Traditional aspects of classification of states

\begin{tabular}{lccc}
\hline & form & volume & $E_{b} \leftrightarrow E_{k}$ \\
\hline solid & unchanged & unchanged & $E_{b}>E_{k}$ \\
liquid & changing & unchanged & $E_{b} \approx E_{k}$ \\
gaseous & changing & changing & $E_{b}<E_{k}$ \\
\hline
\end{tabular}

Table 2 Aspects of classification of states: contact, internal order and movement

\begin{tabular}{lccc}
\hline & Contact & Internal order & Movement \\
\hline solid & exists constantly & exists constantly & $\begin{array}{c}\text { in fixed cell heat } \\
\text { movement }\end{array}$ \\
granular & exists constantly & $\begin{array}{c}\text { is rearranged by } \\
\text { forces; exists in } \\
\text { balance }\end{array}$ & $\begin{array}{c}\text { no movement in } \\
\text { balance }\end{array}$ \\
liquid & exists constantly & does not exist & $\begin{array}{c}\text { sliding over each } \\
\text { other }\end{array}$ \\
& does not exist, & is not & $\begin{array}{c}\text { inertial motion } \\
\text { between two } \\
\text { there are }\end{array}$ \\
& collisions & interpretable & collisions \\
\hline
\end{tabular}

Another body can be interpreted based on mechanical behavior: the ensemble of granules or a granular (or heaped) body. This is a collection of solid bodies in contact. Characteristics of the granular body are given in the second row of Table 2.

\subsection{Movement of the particles forming the medium and their relative arrangement}

The motion forms of the particles forming various medium are well-known in principle see e.g. [27], [21] or [22]. We will briefly summarize the various typical forms of motion and arrangement of particles to describe the conclusions.

\subsubsection{Gaseous body}

Gaseous body is characterized by not having its own shape, a closed container must be used to designate the amount required for "separating" the gaseous body that we wish to examine. A gaseous body exerts pressure on the wall of the container as a result of motion by the particles constituting the gas. The mechanical state of the gaseous body in equilibrium is characterized by the changes of its volume and pressure.

Particles constituting the gas move freely (within the gas) and occasionally collide. The motion of a particle is "inertial" between two collisions. The motion within gas "in a state of rest" is disordered: particles constituting the gas "scatter" on each other upon collision, order cannot be observed in the motion. Moreover, there is no "position" of particles relative to each other, because the relative to each other positions of particles change after every collision. Ordered motion of particles constituting the gas can be observed when the whole of the gaseous body flows, or flow occurs in one part of the gaseous body. Order with good approximation corresponds with the unchanged order of particles, constituting the gas, relative to each other. In reality, this is only the case with good approximation, because there is transverse diffusion beside the flow as well as the change of the diameter of cross section forces the particles to change their orders relative to each other.

\subsubsection{Liquid body}

Liquid body is characterized by not having its own shape; in order to examine, it is required to "separate" the amount using a container with an open top or perhaps a closed one we wish to examine as a liquid body. A liquid body exerts pressure on the bottom and side walls of the container as a result of its weight. The pressure distribution in a liquid body in a state of equilibrium is determined by the height of the liquid column.

The particles constituting the liquid body move freely (within the liquid body) with one condition: all particles are in contact with particles in their close environment at all times. That is to say that, particles constituting the liquid in a "state of rest" are in contact, but their orders are not fixed but also change continuously. On account of continuous contact, we do not speak of liquid particle collision (although the Brownian-motion of solid particles in the liquid is created by exactly the collision of liquid particles). The motion on liquid in a "state of rest" is disordered. The relative position of particles constituting the liquid is no fixed, the positions are continuously changing. Ordered motion of particles constituting the liquid can only be observed when the whole liquid body flows, or flow occurs in one part of the liquid body. Order with good approximation corresponds with when the relative order of particles constituting the liquid is unchanged. In reality, this is only the case with good approximation, see the note made in case of the gaseous body.

\subsubsection{Granular body}

Granular body is characterized by not having its own shape; in order to examine, it is required to "separate" the amount using a container with an open top or perhaps a closed one we wish to examine as a granular body. A granular body exerts pressure on the bottom and side walls of the container as a result of its weight. The internal distribution of forces in a granular body is not primarily determined by the shape of the granular body but by the shape 
of the granules and the relative arrangement of granules (and of course of the weight of the particles), see e.g. [28]. Formally, a granular body is similar to liquid, but the motion of particles constituting the body and the nature of their connection is different in case of a granular body. The granular body, unlike liquid, is not flow in all direction on a horizontal surface but creates a heap.

Particles constituting the granular body do not move (within the granular body) in equilibrium: all granules are in contact with granules in their immediate environment at all times. That is to say that, granules constituting the granular body are in contact, and their orders are fixed. Therefore, the granular body "in the state of rest" is without motion. The motion of particles constituting the granular body can only be observed either if external force acts upon the granular body and granules shift and/or rotate in the "cell" set by granules constituting their environment, or the set of granules or one part of it becomes rearranged. Motion in a granular body, that is to say rearrangement, is ordered if the granular body flows or flow occurs in one part of the granular body. Order with good approximation corresponds with when the relative order of particles constituting the granular body is unchanged. In reality, this is only the case with good approximation; we can argue similarly as in the case of gases and/or liquids (see e.g. emptying of a silo).

\subsubsection{Solid body}

The solid body is characterized by having its individual shape, it is required to "separate" the amount from the matter (has to be cut, or sawn) we wish to examine as a solid body. A solid body only exerts pressure on the container wall fitting to it "from below" and "from sides" (the body is thought to be bricked body), if pressure acts upon the solid body from the open "upper" surface (and balancing pressure occurs on the lower surface). The mechanical state of the solid body is characterized by the change of its shape.

Particles constituting the solid body do not move (within the solid body) from a practical aspect: all particles are in "contact" with particles in their immediate environment at all times. That is to say that, particles constituting the solid body "are in contact" and their orders are fixed. On account of continuous contact, we do not speak of particle's collision. A disordered motion can be interpreted in a solid body "in the state of rest": particles constituting the solid body perform a disordered motion, thermal motion in a "cell" set by particles constituting their environment. The relative position of particles constituting the solid body is fixed. Ordered motion of particles constituting the solid body can only be observed when the whole solid body "flows" (e.g. it shifts and/or rotates like a rigid body), or well-specified layers (e.g. planes of atoms in a crystal structure) perform independent motion (e.g. optical vibrations in the crystal structure) relative to other layers (atomic planes parallel to the planes of selected atoms) and finally if the motion of particles constituting the solid body is collective, continuous (for example tension-compression, bending).

\subsection{Classification of motions}

Motions of the particles forming the body are classified into two main groups: disordered and ordered (collective). We refer to both disordered and ordered motion either to a group of particles or to all the particles (forming the body).

We speak of disordered motion if a group of particles or all particles constituting the body can be selected for which the motion of particles cannot be described by topological tools: the motion of particles constituting the group changes from particle to particle, and there is no topological (that is to say continuity) relationship in this change: the motion of particles in the small environment of a particle with a specific motion essentially differs from the motion (in direction and magnitude) of the specific particle. The position of particles can be variable, or it can be fixed. The position of particles is variable for example in gas and in liquid "in equilibrium", or in the case of a granular body in the development of the equilibrium state accompanied by process of the rearrangement of granules. The position of particles is fixed for example in a crystal structure during the disordered vibration of atoms, or in a granular body in the development of the equilibrium state which process is not accompanied by the arrangement of granules but rather by the changes of granule positions in the cell set by granules constituting their environment. The disordered motion will be referred to as discrete motion hereinafter.

We speak of ordered (that is collective) motion if a group of particles constituting the body can be selected for which the motion of particles can be described by topological tools: the motion of particles in the selected group, in a topological sense, is continuous. In general, the motion of the whole body is ordered if all selectable groups of the particles constituting the body (in topological terms: all environments of all particles) performs an ordered (that is to say continuous in topological terms) motion. The relative position of particles can only be fixed fundamentally, 
as in a solid body, or in the flowing gas, liquid and granular body. The "statement" applying to the relative position of particles in case of flowing particles (i.e., the motion is ordered, that is to say continuous) is only valid for one type of motion: the description provides the motion of particles on a continuous streamline. In reality the motion of particles comprises of ordered motion and motion perpendicular to that on the streamline (see e.g. [15]). At the same time, the existence of a velocity component perpendicular to the tangent of the streamline is not included in the theory (ibid). (Elastic) Deformation and (elastic) vibration occurring in the whole solid body will be referred to as continuous motion, while the laminar motion of flowing bodies will be referred to as quasi-continuous motion.

The motion connected to the cells is such a motion that a) periodically arranged cells (e.g. blocks, prisms with square basement, layers between two planes), b) movement of the elements in the cells can be attached to geometrical formation (e.g. the whole cell moves or rotate as a rigid body, the form of the motion of the element in the cell can be regarded as elongation, shear, bending, torsion etc. - these form give elements of the geometrical formation), c) the motion in the every cells of the whole body can be attached to the same geometrical formation. The motion connected to the cells is called patterned motion. The patterned motion can be discrete (in the neighboring cells the patterns are the same but in one cell the left, in the other one the right variations appear), or continuous (in the neighboring cells the patterns are almost the same, the difference exists in metric, the value of it is small). For example the disintegration of laminar flow into vortex and turbulent flow, or "the third" layer of atoms performs a vibrating motion between the two not moving layers of atoms in the solid body consisting of periodically arranged particles (see e.g. the optical vibrations of the crystal structure, or the different forms of deformations and oscillation of the rigid body built by complex cells [2,3]). In this latter case, the position of particles constituting the body can only be fundamentally fixed, as in the solid body. In the case of flow, the requirement for the fixed position of particles is only met on average; see the condition formulated for the collective motion of flow. The pattern of movement is usually regular in case of crystal structures, but in case of vortex flow the pattern is normally not completely regular, transitional zones can be observed between the individual vortices; the "pattern" is irregular rather than regular in case of turbulent flow. The vortex and turbulent flow will be referred to as quasi-patterned motion collectively.
Consequently, we separated three forms of motion based on the interpretation:

- the motion is disordered, or discrete,

- the motion is ordered (collective), that is topologically continuous, or quasi-continuous,

- in the periodically arranged cells, the motion is characterized by beforehand determined formation, that is patterned, or quasi-patterned, both can be discrete and continuous.

\section{Models of the bodies based on motions of particles constituting the body \\ 3.1 The body}

Interpretation of the body: The body is a collection of more particles in which the particles retain their physical and chemical sameness, move collectively acting upon each other, although the comparative arrangement (i.e., order) of individual particles can change. In general, the body fills a closed domain of the Euclidean space, and the domain is usually associated with various mechanical characteristics, primarily with mass distribution and the ability to resist the specific effect. At the same time, the mechanical effect does not necessarily have to be interpreted as the continuous mapping of a closed domain of the Euclidean space from a mathematical aspect.

According to the interpretation above, gaseous, liquid, granular and solid matters are bodies.

We will give the position of a body made up of a great many particles by providing the position of all points (continuous position vector) of the closed domain modelling it. At the same time, this model does not possess information on where the (physically existing) particles constituting the body are located within the closed domain modelling the body at that moment and what forces act upon them and what their velocity or acceleration is.

\subsection{Models of bodies}

\subsubsection{Introduction}

Body models - solid and rigid bodies, material points, liquids and gases - are commonly known (see, for example, [21] or [22], [27]). Models of bodies can be "redefined" from the point of view of a topological approach [26]. At the same time, it can be specified the type of point set that reflects the character of the body (i.e., the set of points with which the models of the bodies are constructed from a geometric point of view), and can be separated mathematical mappings for which the model is invariant (i.e., the model retains its interpretative properties under the effects on the body). 


\subsubsection{Models of solid bodies}

Interpretation of the solid body: A body is regarded solid, if the relative arrangement (or order) of any two particles remains the same under (mechanical) effect upon the body (and even after its cessation). Geometric model of solid body: In general, solid body is interpreted (modelled) as a closed domain of the Euclidean space. The invariant map from a solid body point of view: the result of (mechanical) effect on the body can be interpreted as the continuous (topological) mapping of a closed domain of Euclidean space for its another closed domain.

Notes:

1. The fact that the topological arrangement remains allows the application of continuous mapping, see e.g. $[1,4]$.

2. Disordered (thermal) motion also exists during the motion of a solid body, but this is restricted to the cells selected by the surrounding particles (atoms) in case of all particles (atom), and the particles (atoms) do not rearrange.

3. The deformable solid body, according to its definition, is such a body in which the order of particles (atoms) constituting the body is fixed, and this fixed order does not change neither during uploading and downloading. Accordingly, only the elastic solid body is the deformable solid body. Rearrangement of the order of particles (atoms) constituting the body is a flow-like deformation. Deformation can occur in case of the prescribed combination of internal forces (the plastic change of shape), and can occur under any external effect (the viscous change of shape).

4. While keeping the topological order in the solid bodies, some particles, such as, e.g. vacancy, substitutional larger or smaller atoms, and dislocations can move inside the deformable body. In general, various topological defects in the solid body interpret different dislocations and different subclasses of solid bodies, which have different mechanical and physical behaviors (see, for example, [29-31]).

According to the interpretation above solid states of matter are solid bodies.

The position of the solid body is given by the set of continuous position vector of all points of the closed domain.

Interpretation of the deformable solid body: A solid body is considered deformable if the distance between any two particles constituting the body (any two points of the Euclidean space) changes as a result of (mechanical) effect acting upon the body then as a result of the cessation of this effect.
The invariant map from a deformable solid body point of view: Mapping of a closed domain of the Euclidean space for another closed domain is continuous. Moreover, or in line with this, to be more precise, we use the changes in metric conditions to describe the changes in distance between the particles, see e.g. [7, 8]. We characterize the deformable solid body with its mass distribution, and with the point-by-point changes of deformation (changes of metric tensor) that occur during the deformation. Internal forces, stresses, arise in the deformable solid body.

Physically all solid bodies are deformable ones.

Interpretation of the rigid body: The rigid body is such a (simplified) model of the deformable solid body, in which the distance between any two points of the body in the model is constant. The invariant map from a rigid body point of view: the (rigid body) motion of the Euclidean space. The position of the rigid body occupied in the Euclidean space is given by the position of one reference point of body and the orientation of the body, that is to say the directions of the axes of the reference trihedron connected to the reference point. In general, mass point is chosen as the reference point of the rigid body, and (one) trihedron assigned to the mass point belonging to the principal inertia is chosen as reference accompanying trihedron. The rigid body is characterized by the total weight concentrated into its reference point, and secondary moment of the mass distribution associated with axes of reference trihedron. No internal force arises in a rigid body.

The kinematically determinate deformable body can be regarded as a rigid body during all such mechanical examinations from the aspect of body behavior, which are not aimed at determining the deformation and the material distribution does not essentially change during the examination. Earth can be considered as a rigid body during its revolving around the Sun, while Earth has to be considered as a deformable solid body in order to determine its flatness or to examine how seismic waves propagate in it.

Interpretation of material point: The material point is such a (simplifying) model of the deformable solid (may be any) body, in which information on the dimension and orientation of the body is disregarded. The position of the material point occupied in the Euclidean space is given by the position of one reference point of the deformable solid body. We generally choose mass point as a reference point in case of a material point. The material point is characterized by the total weight concentrated into the reference point of the deformable solid/rigid body. No internal force arises in the material point. 
Interpretation of the granular body is interpreted in two "steps":

1. Interpretation of the rigid or deformable solid granule: Commonly, a convex-shaped, rigid or deformable solid body is referred to as rigid or deformable solid granule. We characterize the granule by the displacement of its reference point and the rotation of the granule around its reference point as well as deformation of its shape.

2. Interpretation of the dry (consisting of rigid or deformable solid granules) granular body: The ensemble of granules being in constant (in time) contact is considered as dry granular body consisting of rigid or deformable solid granules. We do not apply the continuous mapping of a closed domain of the Euclidean space for another closed domain to kinematically describe the granular body. The mapping of individual granules can in fact be regarded as the continuous mapping of a closed domain but this applies to each and every granule individually and no to the entire domain occupied by the granules. We characterize the granular body by the reference point's displacement of granules, the rotations of granules around own reference points, and change of shape of granules. Internal forces arise in the granular body: in the contact points of two-two granules in contact, or the contact point of the granule and the (solid) constraint surface supporting it. Internal force does not arise in the rigid granule, but internal force does arise in the deformable solid granule.

In this interpretation, the pebbles, sand, cement, flour put into a pile or pumped in a pipe constitutes a granular body.

Notes:

1. To clearly define the balance of the conglomeration of nearly the same size rigid granules, the hypothesis of the three-point support in space must exist; the hypothesis of the two-point support is sufficient in plane $[28,32]$.

2. If the granules are not in contact, e.g. pebbles lying on the ground or solid "micro-satellites" revolve around a planet then these granules do not form a medium (body).

3. To define a flowing granular body, the granules need to be in contact with each other. The ensemble of granules, independent of each other, transported by a carrier medium is not a medium; the medium is what carries the granules. The carrier medium can be liquid (the water of the stream carries the sand floating in the water, or rolls the pebble on the bed of river), but it can also be gaseous (air carries the sand, the dust, and the solid granules smaller than it).
4. The dry adjective refers to the fact that the surface of the granules is not covered by liquid, for example water, or some kind of "glue". Contact force, possibly friction force arises in the dry granular body. Complex contact forms in case of granules that are in contact through the liquid membrane covering the surface of the solid granule, which is not sufficiently modelled with one force in the contact point. Dry sand or gravel are examples of dry granular body, silt and clay are examples of "granular body" contacting through a liquid membrane (surface water film). It is commonly known from soil mechanics (geotechnics), that dry sand or gravel is medium that falls apart to independent granules, while silt and clay are medium that congeal into continuous materials (unless they dry, because then they become flying dust).

5. All granules in the granular body in equilibrium are motionless. Granules are in motion in a flowing granular body.

6. In case of a set consisting of a great number of granules, the "stuck in" granular body can be considered as solid body, while the set of flowing granules as liquid.

\subsubsection{Models of liquid bodies}

Interpretation of the liquid body: A body is considered liquid, if the particles constituting the body that can be considered as a deformable solid body are in continuous motion, and the particles are in continuous contact with each other during continuous motion. A deformable solid container (vessel) or pipe system (river bed, channel, and pipe network) is required to keep the liquid body together; container or pipe system can be open or closed from above (in the opposite direction of gravity). In cases, a kinematically indeterminate solid vessel or pipe can also be sufficient.

In general, liquid body is interpreted (modelled) as the closed domain of Euclidean space enclosed by the deformable solid vessel or pipe system and maybe a free surface of the liquid. Force (introduced in the case of solid bodies) does not directly affect the liquid body, but either the walls of the vessel move and the cross-section of the pipe system changes, or we act upon it through a solid surface. We do not speak about the continuous mapping of a closed domain of the Euclidean space onto another closed domain of it. The liquid is characterized by the mass, velocity, and in cases the angular velocity of the components constituting it. The essence of the liquid state is that the components are in contact and are in constant motion. We do not interpret the force among the individual components 
according to particles in the liquid, but we do interpret the pressure of the whole liquid and in some cases the tension associated with the viscous state.

Notes:

1. The continuous changes of the topological arrangement, or rather the lack of topological arrangement is what does not allow the application of the (continuous) mapping of the Euclidean space.

2. Particles of a liquid in equilibrium are in a disordered motion.

3. In case of a liquid body flow, the collective motion of particles becomes primary and the "collision" of particles becomes secondary. So it is liquid considering its state of matter, while it is a flowing set of particles constantly in contact with each other considering its behavior. In this case, we apply the continuous mapping of a closed domain of the Euclidean space onto another closed domain for both the kinematic (velocity field), and the dynamic (pressure, and tension) characterization.

4. Several forms of motion exist during the flow of liquids:

- ordered motion of particles, that is the flow,

- disordered motion of particles in the flow tube (thermal motion), wandering among flow tube (diffusion),

- rearrangement of particles in case of changes in flow cross-section.

The last of them is imagined as follows. The flowing liquid medium fills the provided cross-section. So if the cross-section of the pipe/vessel doubles or reduces to its half, the flowing liquid medium can only fill it, if the particles of medium are rearranged (and slow down or accelerate). The material does not become continuous since the relative arrangement of particles constituting the medium does not remain constant, and it also changes continuously during flow. Consequently, liquid medium can follow the significant change of cross-section area or form if the order of particles constituting the medium changes: those particles that fell into the same cross-section prior to the cross-section change, will be before-behind each other following the change in the cross-section.

There is an interaction between the particles that make up the liquid. The shape of the free surface of the liquid is determined by this interaction (surface tension). No surface tension occurs in the granular body. Because of this, the liquid and the granular body can be separated. At the same time, the ensemble of flowing grains can be considered as a flowing fluid.
Liquid bodies are commonly classified based on internal friction. The ideal liquid has no internal resistance, this commonly referred to as dry (Newtonian) water, while liquid with internal friction is called wet (non-Newtonian) water. In both cases liquid can be described as liquid starts to flow in the case of the slightest slope; in other words, the body is flowing under the effect of the constant shear "force".

Such liquids exist, which flow slowly or do not start to flow at all on a slight slope as due to gravity, but the flow, that is to say the internal rearrangement starts under the effect of the slightest pressure. Pastes, varnishes, potter's (prior to burning) clay or raw bread pasta, fresh concrete or mortar are commonly listed here.

Certain liquids have the characteristic that the particles stick together and move together as a solid body. These are elastic liquids. It is clear based on the interpretation that these do not behave like liquids but rather like solid matters specifically during the elastic behavior [33].

The plastic shaping of metals, some types of plastic can be considered "dual" to liquid: certain zones of the solid body behave as liquids [24].

We also speak of flow in case of glaciers and ice floods. The ice flood is not a real liquid, since the order of water molecules constituting the ice flood is fixed. The difference between the flow of water and ice can be described with when the two floods meet: the water of rivers mix, ice floods flow side-by-side. In other words: although ice floods flow but they are not liquids.

\subsubsection{Models of gaseous bodies}

Interpretation of the gaseous body: A body is considered gaseous, if the deformable solid bodies forming the body do not come into contact with each other during their continuous in time movement, but at most they collide with each other. A closed (kinematically determinate, e.g. steal) solid vessel or pipe system is required to keep the gaseous body together. (In cases, a kinematically indeterminate solid vessel or pipe can also be sufficient.)

Note: External force (e.g. gravity) changes the density distribution in the gas and can also plays the role of the vessel.

In general, the gaseous body is interpreted (modelled) as the closed domain of Euclidean space enclosed by the deformable solid vessel or pipe system keeping the gas together. Force (introduced in the case of solid bodies) cannot be interpreted in case of the gaseous body, mechanical effect can be triggered by moving the walls 
of the container (e.g. piston), and by solid bodies moved within the gaseous body. In the case of gas the continuous mapping of a closed domain of the Euclidean space onto another closed domain does not reflect the continuous colliding of particles of gas.

Notes:

1. In case of gas, the continuous changes of the topological arrangement, or rather the lack of topological arrangement is what does not allow the application of the (continuous) mapping of the Euclidean space.

2. Particles of a gaseous body in equilibrium are in a disordered motion. It is usually assumed that the particles collide with each other.

3. In case of a gaseous body flow, the collective motion becomes primary and the "collision" of particles becomes secondary. So it is gas considering its state of matter, while it is a flowing set of particles considering its behavior. In this case, similarly to liquid, we apply the continuous mapping of a closed domain of the Euclidean space onto another closed domain for primarily the kinematic characterization and secondarily to describe the pressure arising in gas.

4. Several forms of motion exist during the flow of gas similar to fluid flow:

- ordered motion of particles, that is the flow,

- disordered motion of particles in the flow tube (thermal motion), wandering among flow tube (diffusion),

- rearrangement of particles in case of changes in flow cross-section.

In the latter case, in principle, a comment similar to that of a liquid can be made. So if the cross-section of the pipe/vessel doubles or reduces to its half, or shape changes greatly than the mean free path of a particles of gaseous medium doubles or reduces to its half, but the gaseous body itself does not become continuous since the relative arrangement of particles constituting the medium constantly changing irrespective of the size and shape of the cross-section.

According to the interpretation above, a material enclosed in a container in a gaseous state of matter is a gaseous body. An ensemble of "shaken", with significant kinematical energy particles, colliding with each other, can be considered as a gaseous substance. A set of solid particles revolving around a planet e.g. rings of Saturn and Uranus cannot be considered as gases from this aspect. Similarly to this, the mechanical state of matter of the rare interstellar dust in the space is not determined by the collisions of interstellar dust's particles therefore it cannot be considered as a gaseous body.
The gaseous body is characterized by the mass, velocity and "continuous" collision of the particles constituting the gas. We do not interpret "unique" forces arising during the collision of particles in gas, but we do interpret the pressure exerted by gas onto a surface.

It is no coincidence that we named the kinematically determinate deformable solid vessel as the "body" holding the medium together. For instance, if the vessel is a kinematically indeterminate tarpaulin, then no pressure arises with the excessively small kinetic energy, that means that particles forming "gas" do not rebound: the wall of the "vessel" absorbs the kinetic energy of particles colliding into it, and the motion of the particles ceases. An example for this is the "air-tight" canvas sack, which is not inflated: there is air in the sack but the atoms/molecules and the velocity of them are not suitable for stretching the wall of sack. (Stretching makes the kinematically indeterminate solid body into kinematically determinate solid body, that is to say suitable for taking up internal pressure.)

Gas is commonly distinguished as thick and rare gas according to the collision of particles constituting the gas. We commonly speak of thick gas in case of particles colliding with each other. We speak of rare gas when the particles only collide with the wall of the vessel.

\section{Mathematical description of disordered, collective and patterned motion}

\subsection{Disordered motions}

Continuous mapping of the Euclidean space cannot be used for describing the disordered motion occurring in a closed domain of the Euclidean space. We can choose from two types of methods based on the number of particles.

The case of the number of particles trackable by computer technology: We track the motion of each and every particle. We can apply this during the examination of lattice vibrations [34], and also in case of the equilibrium of rigid granules (see e.g. [28, 32]). The discrete element method is suitable for the modeling of the random arrangement of the particles and the deformation of the granules in the grain set, see e.g. $[35,36]$. To describe the flow of particles with a description of each particle state, the theory of cellular automata can be used (the phenomenon to be modelled is to leave a finite amount of particle through a narrow opening) [37].

The case of the number of particles not trackable by computer technology: In case of disordered movement, instead of tracking the individual particles, we determine the average behavior of particles. We can do this in many 
ways (see, e.g. [38]); which basically delimits four options; we do not list the case second, hydrodynamic approach, here, and extend the phenomenological method using internal variables).

1. Only macroscopic features can be considered. This leads to equilibrium (phenomenological) thermodynamics (see e.g. [39-42]). In this case, there is no information on the movement of the particles forming the system, the system is considered homogeneous. For the analysis of inhomogeneity, we interpret internal time-dependent processes (non-equilibrium phenomenological) thermodynamics, [43-45]), when changes within the system are defined as a function of spatial coordinates and time. In addition to inhomogeneity, we can also describe the internal structure and its change. This can be done in part by using the extended thermodynamic approach (see e.g. [46]), partly by using internal variables (see e.g. [47, 48]).

2 . The disordered motion is modelled by random motion. This assumes that the particles that make up the system move and collide. We consider the distribution of the direction and velocity magnitude of the motion random. We verify the assumptions related to distribution by experiments: this is the kinetic theory of gases (see e.g. [39, 43, 45, 49-51]).

3 . We consider the system itself or the distribution of the particles that form the system to be a probability variable. This leads to statistical discussion, to statistical mechanics (generally to statistical physics) (see e.g. [39, 43, 45, 52-55]).

\subsection{Ordered, or collective motions}

\subsubsection{The material is considered to be continuous, homogeneous and without internal structure}

The model can be applied to two media. One is a body made of laminar flowing particles (gas, liquid, granules) and the other is a deformable solid body.

The theory of laminar flowing bodies leads to chapters of the theory describing the hydrodynamic laminar flow $[9,10,15]$.

The solid body theory leads to the theory of classical continuum (i.e., the theory of elasticity) [7, 8].

The continuous mapping of the Euclidean space can be used to describe the ordered motion occurring in a closed domain of the Euclidean space. During this we fundamentally disregard the actual number of the particles and we do not examine the motion of the particles but the particles "distributed" in the Euclidean space, e.g. we analyze a bounded and closed domain of the Euclidean space instead of a system of particle. In this modelling the next step is to paraphrase the elements of Newtonian mechanics concerning material points (see e.g. [56]) for a continuous distribution bodies (see e.g. [13]). While forces in mechanics of system of material points can be linked to the distances between material points, internal force in mechanics of continuous system of material points will be proportionate to the change the distances between of two points of the continuous medium. (The force must also be interpreted according to the example of the Newtonian force but in a different form, regarding the continuous medium; see the definition of stress $[7,8]$ ).

\subsubsection{The material is considered to be continuous, inhomogeneous and/or having internal structure}

The inhomogeneity of a substance considered to be continuous can be investigated by probabilistic modeling of inhomogeneity, heuristic modeling of the internal structure, functions having continuous domain of definition over discrete internal structure, and introduction of variables interpreted in the tangent space of particles.

The inhomogeneity of material properties for the test is defined as the distribution of material properties as probability variables [57-60]; during this, the theoretical framework of the classical continuum is maintained. The inhomogeneity is "smoothable"; the procedure refers to "averaging", "smoothing" and homogenizing of the state operators [61].

For examination of the inhomogeneity as an internal structure of the material, besides the displacement other kinematic characteristics (rotation, angular change, elongation, and deformation) are also considered at every point of the Euclidean space.

The first step towards generalization was made by the Cosserat brothers, who, similarly to the rotation of Darboux frame on a curve as well as on a surface considered the rotation of Darboux frame of the three-dimensional Euclidean space as an independent kinematic freedom. No experimental background was available to build the model; they assumed the structure of elastic energy, and the equations of theory were derived using variation principle [62]. Fifty years after the work of the Cosserat brothers, the generalization of the classical continuum was generally assumed to be possible by interpreting a continuous internal kinematic variable. There was no experimental background for model building in this case; usually, the method of derivation of the equations of theory by analogy to the procedures used in the classical continuum was only applied (see, for example, most of the studies in [63]). The theory that only uses the concept of rotation and stress moment leads to the micro-polar continuum [64]. 
The assumption of different internal structures - matrix and granules sitting on it, although there was still no experimental background for model building; by using methods developed by analogy to the procedures used in the classical continuum, various micro-continuums can be interpreted and the formulae of the theory can be deduced (see e.g. [65]); for generalized continuum see also [66-70]). The correlations based purely analogue derivation contain inconsistencies [71], in addition, a number of material constants occur in theory, but experimental data are not known, and even the nature of attempts to determine material constants is unknown (see e.g. [64, 65]).

For the inhomogeneity of the internal structure of the material, the body is divided into finite cells for examination, while the domain of the variables assigned to the cells is discrete, but the internal structure of body is described with functions with continuous domain of definition (see e.g. [2, 3]). Theories thus obtained describe the states of non-continuous systems, theories themselves being considered to be a numerical method (see introductory chapters in the book in $[2,3]$ and $[4,72])$. Kunin, just like the Cosserat brothers, does not rely on experiments, but derives the theory from the presupposition of the elastic energy of the discrete internal structure of material by analogy and the assumption of the minimum of elastic energy [2, 3]. The method explains (using series expansion of state-determining functions) why many material constants occur, and at the same time that they should not be measured, but determined during the construction of the theory (ibid).

For the inhomogeneity of the internal structure of the material, the tangent space of each point of the body can be ordered with different degrees of autonomic kinematic (and other non-mechanical content) variables [73]. These degrees of freedom - in the sense of $[2-4,72]$ - should be interpreted as elements of series expansion in the field of functions with continuous domain, rather than functions of functions with discrete domain.

Note: in the case of the probability variable we use the method of the classical continuum, the other three approaches are the outline of the classical continuum: it is a description of a discrete system rather than a continuous one (see Subsection 4.3).

\subsection{Patterned motions}

The model can be applied to two media. One is a deformable solid body consisting of periodically arranged rigid particles, and the other is a (gas, liquid, granular) body flowing in a pattern (i.e., non-laminar). Both are interpreted as a numerical method: base functions and error vector are selected; we use error principle to determine to solve the equations (the elements of numerical method see e.g. [74]). The relationship among the periodically arranged deformable solid body, the numerical method and the generalized continuum is illustrated by the following thought. The region of the examined solid body composed of bound particles must be partitioned on periodically arranged cells to define patterned motion. The periodically arranged cells are usually taken in form of brick, but can be taken in form of prism, or of disk. There are one or more (e.g. 8, 27 etc.) periodically arranged particles in the brick-cells, one or more (e.g. 4, 9 etc.) periodically arranged line of periodically arranged particles in the prism-cells, one or more (e.g. 2, 3 etc.) periodically arranged layer of periodically arranged particles in the disk-cells. The form of motion must be defined, as it was mentioned above: rigid body translation and/or rotation, elongation, shear, bending, torsion, or something else. The motions in different cells are independent each of other, that is the motions in the cells are fundamentally discrete. The forms of motion inside the cells are usually called by internal kinematic variable. The domain of definition of the functions describing these motions is discrete point, usually the reference points of cells. The motions inside the cells are regarded continuous function as in time as well as in space. The functions with discrete domain of definition must be approximated by series expansion in terms of functions with continuous domain of definition to create continuous in space model/ theory. (Note: Partial differential derivatives of unknown state functions in the theory are also considered as internal variables.) Two further steps are required for establishing the continuous in space model/theory: the error vector and error principle must be interpreted in the space of (continuous) functions with continuous domain to determine the approximate solution (in the space of functions with discrete domain of definition) (see e.g. [2-4, 72]). In practice, some kind of principle of variation (which prescribes the minimum of action or entropy production) is used. For details in each case, see also Section 4.2.2 below.

The relationship between the theory pattern movement, that is non-laminar flow (eddy or turbulent flow, see e.g. $[9,10,15]$ as well as [75]) of body consisting of free particles (gaseous, liquid and granular body) and numerical methods is illustrated by the following thought. In case of vortex flow, the velocity field of a specific vortex flow is considered as given in the entire flow space (these are the "base functions"), and the velocity field is produced by the 
integral of these flow images (see e.g. $[9,10])$. Accordingly, the interpretation of error vector is omitted. (Note: The theory does not deal with how the two vortex flows effect each on other.) The turbulent motion can be interpreted as a loss of stability. During this, the increase of velocity leads to repeated periodic loss of stability. According to this newer and newer forms of motion occur, which finally ("in the limit") lead to the fully developed turbulence (see e.g. [15]). The application of series expansion and keeping the first member in the series (ibid) mean exactly the application of base functions, the criterion of loss of stability can be considered as the error principle. The interpretation of turbulence can be based on the interpretation of characteristic distance in a turbulent liquid, the examination of energy dissipation of flow and the random interpretation of energy dissipation too. The method comes from Kolmogorov, for detailed description see e.g. [76]. The Naiver-Stokes equation, as the equation describing flow provides the differential geometry background, which describes the ordered (flowing) motion. The disordered that is to say different from the ordered flow image but superimposed, the energetic "projection" of forward-backward flow motion which is partially perpendicular in direction to the flow and partially identical with it, or the energy dissipation interpreting the effect of turbulent (that is to say swirling) motion as a random variable. The "loss" of energy required for maintaining the flow, that is to say the dissipation of energy also has to be considered in case of turbulent flow. Within the characteristic distance (based on the three applied hypothesis) the Naiver-Stokes equation possesses the symmetric characteristics that the behavior characterized by distribution applied for the motion is self-similar. The three hypotheses for describing dissipation is equivalent with the prescription of base function characteristics.

The turbulent flow during its modeling is divided into a laminar flow and turbulent flow zone (see e.g. [77]). The numerical modeling of the turbulent flow is primarily done by the "Direct Numerical Method" (see e.g. [78]), where the either "Divergence Free Method" or "Vortex Method" is used (ibid). The "Large Eddy Simulation" [79], which is based on the "Subgrade-scale" model, is widely used to describe turbulent flow [80]. One of the basic problems of modeling turbulent flows is the so-called closure problem: the Navier-Stokes equation refers to the averaged "mean part" in which the term "fluctuation" is in nonlinear form; the task is to choose the right algebraic or differential expression [81-83]. The turbulent flow is associated with heat flow and energy dissipation, flow characteristics are broken down into "mean flow" and fluctuation, and the ergodic hypothesis is assumed (see, for example, [83-85]).

Note: The patterned motion of the deformable solid body built by periodically arranged rigid particles can be continuous (quasi-continuous) or discrete. Slow change according to spatial coordinates across cells is continuous (quasi-continuous), rapid change leads to discrete motion. Usually, both are described by continuous functions according to the spatial coordinates across the cells, but the rapidly changing movement is not continuous, because this motion cannot be described by continuous mapping of a domain of the Euclidean space to another one. In fact, not the motion is continuous, but the domains of definition of the functions used to describe the motion are continuous (since the functions with the discrete domain of definition have been approximated by series expansion in the field of functions of continuous domain of definition).

The quick change of the patterns in the neighboring cells gives the discrete (global) motion, the slow change of the patterns gives the continuous (quasi-continuous) motion. Discrete layouts are not automatically returned by functions with continuous domain of definition. As part of the investigation, a solution consistent with a discrete domain of definition should be produced from the continuous solution. At the same time functions with continuous domain of definition provide (mathematically and) mechanically interpretable result only in discrete locations (for solid bodies, see, for example, [2, 3]).

\section{Conclusions}

The paper focused on the characterization of the mechanical behavior of matter based on the motion of particles constituting the matter. The distinction of bodies founded on the states of matter is based exactly on this: gas is an ensemble of colliding particles, liquid is an ensemble of particles in continuous contact, but these do not maintain their order, and finally the solid body is an ensemble of particles in a fixed order. We have shown that the interpretation of the state of the body based on the aspect of the shape/volume conservation/change, ratio of the bond and the kinetic energy dependent on the heat movement, and finally the contact of the particles that make up the material, existing internal order and movement of particles leads to the same classification of bodies.

We have shown that the motions of particles constituting the medium can be classified into three distinct groups: disordered, collective and patterned motion. In 
the presence of a small number of particles, the status of each particle is monitored separately (grain set, discrete element method). The presence of a high number of particles does not allow the direct application of the apparatus of the Newtonian mechanics for every particle, in some sense distribute; "continuous" model must be applied. The "distribution" must be in accordance with the motion of the particles constituting the body. We interpret three differently "distributed" systems for three different forms of motion. We assign statistic distribution for the disordered motion of free particles (gas). To describe the collective movement of the free particles (e.g. gas $\rightarrow$ laminar flow) and bound particles (e.g. solid $\rightarrow$ deformation), the all particles are distributed over point set of the domain occupied by particles, and replacing them with a continuous set over which continuous functions are selected to describe the system. In order to describe the patterned motion of free moving particles (e.g. gas), we characterize the whole system by summarizing the local solutions reflecting each pattern. We assign (approximate) functions of continuous domain of definition to the patterned movements of the periodically arranged, fixed-position particles (lattice system) instead of functions with discrete domain of definition. The disordered motion leads to thermodynamic description and to kinetic gas theory and statistical mechanics. Within the thermodynamic description, the without internal structure and the internal variable approach can be distinguished. Collective that is to say continuous motion leads to the laminar flow and the classical continuum. Patterned motion leads to partly vortex and turbulent motion descriptions, and partly to grid continuum. The various models of grid continuum interpret the various generalized continua. In the case of grid continuum, the choice of base functions indicates that the generalized continuum is not a continuous medium, but a theory describing the mechanical behavior of a discrete, periodically arranged medium with functions of continuous interpretation domain. In case of patterned flow and disordered motion - this is the turbulent flow - both apparatus are simultaneously applied for the description of both the ordered and the disordered motion. This is the Kolmogorov-type random approach.

We have shown, see in Table 3 that freezing degrees of freedom of motion interprets a series of models. Reading them from one direction is specialization, and it is generalization from the other direction.

Note: Independent rotation ceases in the model of classical continuum, see e.g. [86].

The forms of motion of the media and the characteristics of the model can be summarized in the Table 4 below. gas $\leftrightarrow$ liquid $\leftrightarrow$ granular body $\leftrightarrow$ grid continuum $\leftrightarrow$ classical continuum $\leftrightarrow$ rigid body $\rightarrow$ material point

Table 3 The conditions for changing the model

\begin{tabular}{|c|c|}
\hline gas: & occasional collision of particles \\
\hline \multicolumn{2}{|l|}{$\uparrow$} \\
\hline liquid: & continuous contact of particles, but there is no fixed order \\
\hline \multicolumn{2}{|l|}{$\uparrow$} \\
\hline granular body: & $\begin{array}{l}\text { the particles are in constant contact, in the equilibrium there is (not necessarily regular, periodic) order of } \\
\text { the particles; as a result of external force, the particles usually rearrange }\end{array}$ \\
\hline \multicolumn{2}{|l|}{$\uparrow$} \\
\hline grid continuum: & $\begin{array}{l}\text { the fixed (usually regular) order of particles remains, although the motion can also be disordered, the } \\
\text { distance and orientation among particles changes arbitrarily }\end{array}$ \\
\hline \multicolumn{2}{|l|}{$\uparrow$} \\
\hline classical continuum: & $\begin{array}{l}\text { the motion is collective beside the fixed order of particles (that is the points of Euclidean space), the } \\
\text { distance among the particles of the body changes, the change is continuous (the particles are distributed, } \\
\text { there is no rotation of point) }\end{array}$ \\
\hline \multicolumn{2}{|l|}{$\uparrow$} \\
\hline rigid body: & $\begin{array}{c}\text { beside the fixed order of particles (that is the points of Euclidean space), the distance among the points of } \\
\text { body is constant }\end{array}$ \\
\hline \multicolumn{2}{|l|}{$\downarrow$} \\
\hline material point: & $\begin{array}{l}\text { the dimension of the body is irrelevant (the modelled body can be solid body but it can also be liquid(drop) } \\
\text { "held together"). (the transition in the inverted direction is not clear therefore the arrow is in one direction) }\end{array}$ \\
\hline
\end{tabular}


Table 4 The forms of motion of the media and the characteristics of the model

\begin{tabular}{|c|c|c|c|c|}
\hline \multicolumn{2}{|c|}{ State } & Equilibrium & \multicolumn{2}{|c|}{ Movement } \\
\hline \multicolumn{2}{|c|}{ Motion of particles } & Disordered & Collective & Patterned \\
\hline \multirow{3}{*}{ Free particles } & Gaseous & collision & \multirow{3}{*}{ laminar flow } & \multirow{3}{*}{ vortex and turbulent flow } \\
\hline & & contact & & \\
\hline & Granular & $\begin{array}{l}\text { no motion: equilibria } \\
\text { arrangement }\end{array}$ & & \\
\hline Fixed particles & Solid & vibration around grid points & shape change & $\begin{array}{l}\text { motion in the cells, optical } \\
\text { and acoustic vibrations }\end{array}$ \\
\hline \multicolumn{2}{|c|}{ Type of theory } & $\begin{array}{l}\text { phenomenological, } \\
\text { internal-variable (extended) } \\
\text { thermodynamics; kinetic gas } \\
\text { theory; statistical mechanics; } \\
\text { balance of granular material }\end{array}$ & classical continuum & $\begin{array}{c}\text { generalized continua: } \\
\text { cell system with rigid or } \\
\text { deformable particles sitting in } \\
\text { the grid points }\end{array}$ \\
\hline \multicolumn{2}{|c|}{ Characterization of description and motion } & $\begin{array}{l}\text { continuous distribution and } \\
\text { discrete motion }\end{array}$ & $\begin{array}{l}\text { continuous description and } \\
\text { continuous motion }\end{array}$ & $\begin{array}{l}\text { description over cells is } \\
\text { continuous, the motion inside } \\
\text { the cells is discrete }\end{array}$ \\
\hline
\end{tabular}

\section{References}

[1] Noll, W. "Lectures in the Foundation of Continuum Mechanics and Thermodynamics", In: The Foundations of Mechanics and Thermodynamics, 1st ed., Springer, Berlin, Germany, 1974, pp. 293-324. https://doi.org/10.1007/978-3-642-65817-4

[2] Kunin, I. A. "Elastic Media with Microstructure I", 1st ed., Springer, Berlin, Germany, 1982.

https://doi.org/10.1007/978-3-642-81748-9

[3] Kunin, I. A. "Elastic Media with Microstructure II", 1st ed., Springer, Berlin, Germany, 1983 https://doi.org/10.1007/978-3-642-81960-5

[4] Lámer, G. "A deformálható szilárd, folytonos közegek matematikai modellezésének egyes kérdéseiről: topológikus, metrikus és numerikus szempontok" (Some questions of mathematical modelling of deformable solid continuous media: topological, metrical and numerical points of view), In: Mérnökgeológia-Kőzetmechanika 2013. Konferencia (Proceedings of Engineering-Rock Mechanics 2013 Conference), Budapest, Hungary, 2013, pp. 317-332. (in Hungarian) [online] Available at: http://mernokgeologia.bme.hu/ocs/index.php/ konferencia/2013/paper/viewFile/33/32 [Accessed: 03 June 2019]

[5] Schouten, J. A. "Tensor Analysis for Physicists", 1st ed., Clarendon Press, Oxford, UK, 1951.

[6] Shultz, B. F. "Geometrical Methods of Mathematical Physics", 1st ed., Cambridge University Press, Cambridge, UK, 1982.

[7] Love, A. E. H. "A Treatise on the Mathematical Theory of Elasticity", 4th ed., Cambridge University Press, Cambridge, UK, 1927.

[8] Lurie, A. I. "Theory of Elasticity", 1st ed., Springer, Berlin, Germany, 2005 .

https://doi.org/10.1007/978-3-540-26455-2

[9] Lamb, H. "Hydrodynamics", 6th ed., Dover Publication, New York, NY, USA, 1952.

[10] Milne-Thomson, L. M. "Theoretical Hydrodynamics", 2nd ed., The Macmillan Company, New York, NY, USA, 1950.

[11] Tonti, E. "The Mathematical Structure of Classical and Relativistic Physics. A General Classification Diagram", Birkhäuser, New York, NY, USA, 2013. https://doi.org/10.1007/978-1-4614-7422-7
[12] Bell, J. F. "Mechanics of Solids. The Experimental Foundations of Solid Mechanics", Springer, Berlin, Germany, 1984.

https://doi.org/10.1007/978-3-642-69565-0

[13] Truesdell, C., Toupin, R. "The Classical Field Theories", In: Flügge, S. (ed.) Principles of Classical Mechanics and Field Theory, 1st ed., Springer, Berlin, Germany, 1960, pp. 226-858. https://doi.org/10.1007/978-3-642-45943-6

[14] Truesdell, C., Noll, W. "The Non-Linear Field Theories of Mechanics", Encyclopaedia of Physics, 25(3), pp. 638-640, 1966. https://doi.org/10.1017/S0022112066210302

[15] Landau, L. D., Lifschitz, E. M. "Fluid Mechanics", 2nd ed., Pergamon Press, Oxford, England, 1987. https://doi.org/10.1016/C2013-0-03799-1

[16] Sarfarazi, V., Schubert, W. "Sliding Phenomena in Intermittent Rock Joint", Periodica Polytechnica Civil Engineering, 61(3), pp. 351360, 2017.

https://doi.org/10.3311/PPci.9466

[17] Wu, Y., Yamamoto, H. "Numerical Investigation on the Reference Crushing Stress of Granular Materials in Triaxial Compression Test", Periodica Polytechnica Civil Engineering, 59(4) pp. 465-474, 2015. https://doi.org/10.3311/PPci.7694

[18] Asszonyi, Cs., Csatár, A., Fülöp, T. "Elastic, thermal expansion, plastic and rheological processes - theory and experiment", Periodica Polytechnica Civil Engineering, 60(4) pp. 591-601, 2016. https://doi.org/10.3311/PPci.8628

[19] Crowe, M. J. "Theories of the Word from Antiquity to the Copernican Revolution", 2nd ed., Dover Publications, New York, NY, USA, 2001.

[20] Simonyi, K. A. "Cultural History of Physics", 1st ed., CRC Press, New York, NY, USA, 2012. https://doi.org/10.1201/b11630

[21] Nagy, E., Beleznay, F., Mayer, T., Szervánszky, Gy. "Fizikai kislexikon" (Physical Little Lexicon), Müszaki Könyvkiadó, Budapest, Hungary, 1977. (in Hungarian)

[22] Wikipedia "State of matter" [online] Available at: https://en.wikipedia.org/wiki/State of matter [Accessed: 04 June 2019] 
[23] Wikipedia "Rheology" [online] Available at: https://en.wikipedia. org/wiki/Rheology [Accessed: 04 June 2019]

[24] Dixit, P. M., Dixit, U. S. "Plasticity: Fundamentals and Applications", 1st ed., CRC Press, Boca Raton, FL, USA, 2015.

[25] Wikipedia "Fluid" [online] Available at: https://en.wikipedia.org/ wiki/Fluid [Accessed: 04 June 2019]

[26] Lámer, G. "Solid and soft body with and without structure", In: Quasi-Static Deformations of Particular Materials, Proceedings of QuaDMP'03 Workshop, Budapest, Hungary, 2003, pp. 159-166.

[27] Budó, Á. "Mechanika" (Mechanics), 5th ed., Tankönyvkiadó Vállalat, Budapest, 1972. (in Hungarian)

[28] Lámer, G. "Erővonalak edényben szabályosan elrendezett golyók halmazában" (Force Distributions in the heap of regularly arranged in pot spheres), presented at XI. Magyar Mechanikai Konferencia, MaMeK, Miskolc, Hungary, Aug. 29-31, 2011. (in Hungarian)

[29] Friedel, J. "Dislocations", 1st ed., Pergamon, Oxford, UK, 1964. https://doi.org/10.1016/C2013-0-02250-5

[30] Hirth, J. D., Lothe, J. "Theory of Dislocations", McGraw-Hill Books Company, New York, 1972

[31] Ziman, J. M. "Models of Disorder: The Theoretical Physics of Homogeneously Disordered Systems", 1st ed., Cambridge University Press, Cambridge, UK, 1979.

[32] Lámer, G. "Szemcsehalmazok egyensúlyának és átrendeződésének az állapotegyenlete" (Equations of heaped body in the state of equilibrium and of rearrangement), presented at XI. Magyar Mechanikai Konferencia, MaMeK, Miskolc, Hungary, Aug. 29-31, 2011. (in Hungarian).

[33] Lodge, A. S. "Elastic Liquids", 1st ed., Academic Press, Manchester, UK, 1964.

[34] Born, M., Huang, K. "Dynamical Theory of Crystal Lattices", 1st ed., Clarendon Press, Oxford, UK, 1954.

[35] Bagi, K. "A diszkrét elemek módszere" (Discrete Element Method), 1st ed., BME Tartószerkezetek Mechanikája Tanszék (Budapest University of Technology and Economics, Department of Structural Mechanics), Budapest, Hungary, 2007. (in Hungarian)

[36] García-Rojo, R., McNamara, S., Hermann, H. J. "Influence of Contact Modelling on the Macroscopic Plastic Response of Granular Soils Under Cyclic Loading", In: Capriz, G., Mariano, P. M., Giovine, P. (eds.) Mathematical Models of Granular Matter, Springer, Berlin, Germany, 2008, pp. 109-124. https://doi.org/10.1007/978-3-540-78277-3

[37] Wirth, E., Szabó, Gy. "Overlap-avoiding Tickmodel: an Agentand GIS-Based Method for Evacuation Simulations", Periodica Polytechnica Civil Engineering, 62(1) pp. 72-79, 2018 https://doi.org/10.3311/PPci.10823

[38] Uffink, J. "Compendium of the Foundations of Classical Statistical Physics", In: Butterfield, J., Earman, J. (eds.) Philosophy of Physics, Part B, Elsevier, Amsterdam, Netherlands, 2007, pp. 923-1074. https://doi.org/10.1016/B978-044451560-5/50012-9

[39] Horváth, J. "Termodinamika és statisztikus mechanika" (Thermodynamics and Statistical Mechanics), Tankönyvkiadó Vállalat, Budapest, Hungary, 1960. (in Hungarian)

[40] Truesdell, C. "Rational Thermodynamics", 2nd ed., Springer, New York, NY, USA, 1984.

https://doi.org/10.1007/978-1-4612-5206-1
[41] Truesdell, C. A. "The Tragicomical History of Thermodynamics, 1822-1854", Springer, Berlin, Germany, 1980. https://doi.org/10.1007/978-1-4613-9444-0

[42] Matolcsi, T. "Ordinary Thermodynamics", 1st ed., Akadémia Kiadó, Budapest, Hungary, 2004.

[43] de Groot, S. R., Mazur, P. "Non-Equilibrium Thermodynamics", North-Holland Publishing Co., Amsterdam, Netherlands, 1962.

[44] Gyarmati, I. "Non-equilibrium Thermodynamics. Field Theory and Variational Principles", 1st ed., Springer, Berlin, Germany, 1970. https://doi.org/10.1007/978-3-642-51067-0

[45] Landsberg, P. T. "Thermodynamics and Statistical Mechanics", Oxford University Press, Oxford, UK, 1978.

[46] Jou, D., Lebon, G., "Extended Irreversible Thermodynamics", 4th ed., Springer, Dordrecht, Netherlands, 2010 https://doi.org/10.1007/978-90-481-3074-0

[47] Valanis, K. C. "Irreversible Thermodynamics of Continuous Media", Springer, Udine, Italy, 1971.

[48] Verhás, J. "Termodinamika és reológia" (Thermodynamics and Rheology), 1st ed., Müszaki Könyvkiadó, Budapest, Hungary, 1985. (in Hungarian)

[49] Maxwell, J. C. "Theory of Heat", 3rd ed., Longmans, Green and Co., London, UK, 1872.

[50] Boltzmann, L. "Vorlesungen über Gastheorie I" (Lectures on Gas Theory I), 1st ed., Barth, Leipzig, Germany, 1896. (in German)

[51] Boltzmann, L. "Vorlesungen über Gastheorie II" (Lectures on Gas Theory II), 1st ed., Barth, Leipzig, Germany, 1898. (in German)

[52] Gibbs, J. W. "Elementary Principles in Statistical Mechanics", Cambridge University Press, Cambridge, UK, 2011. https://doi.org/10.1017/CBO9780511686948

[53] Ehrenfest P., Ehrenfest, T. "The Conceptual Foundations of the Statistical Approach in Mechanics", Dover Publications, Mineola, NY, USA, 2015

[54] Landau, L. D., Lifschitz, E. M. "Statistical Physics", 3rd ed., Elsevier, Amsterdam, Netherlands, 1980. https://doi.org/10.1016/C2009-0-24487-4

[55] Presutti, E. "Scaling Limits in Statistical Mechanics and Microstructures in Continuum Mechanics", 1st ed., Springer, Berlin, Germany, 2009. https://doi.org/10.1007/978-3-540-73305-8

[56] Landau, L. D., Lifschitz, E. M. "Mechanics", 3rd ed., Elsevier, Amsterdam, Netherlands, 1976.

[57] Kröner, E. "Statistical Continuum Mechanics", 1st ed., Springer, Vienna, Austria, 1971. https://doi.org/10.1007/978-3-7091-2862-6

[58] Weiner, J. H. "Statistical Mechanics of Elasticity", John Wiley \& Sons, New York, NY, USA, 1983.

[59] Lomakin, V. A. "Teoriya uprugosti neodnorodnykh sred" (Theory of Elasticity of Inhomogeneous Media), Izdatel'stvo Moskovskogo Universiteta, Moscow, Russia, 1976. (in Russian)

[60] Sermergor, T. D. "Teoriya uprugosti mikroneodnorodnykh sred" (Theory of Elasticity of Micro-inhomogeneous Media), Nauka, Moscow, Russia, 1977. (in Russian)

[61] Jikov, V. V., Kozlov, S. M., Oleinik, O. A. "Homogenization of Differential Operators and Integral Functionals", 1st ed., Springer, Berlin, Germany, 1994

https://doi.org/10.1007/978-3-642-84659-5 
[62] Cosserat, E., Cosserat, F. "Théorie des Corps Déformables" (Theory of Deformable Bodies), Hermann, Paris, France, 1909. (in French)

[63] Kröner, E. (ed.) "Mechanics of Generalized Continua", 1st ed., Springer, Berlin, Germany, 1968. https://doi.org/10.1007/978-3-662-30257-6

[64] Nowacki, W. "Theory of Micropolar Elasticity", 1st ed., Springer, Vienna, Austria, 1970. https://doi.org/10.1007/978-3-7091-2720-9

[65] Eringen, A. C. "Microcontinuum Field Theories, I. Foundations and Solids", 1st ed., Springer, New York, NY, USA, 1998. https://doi.org/10.1007/978-1-4612-0555-5

[66] Altenbach, H., Eremeyev, V. A. (eds.) "Generalized Continua from the Theory to Engineering. Applications", Springer, Vienna, Austria, 2013.

https://doi.org/10.1007/978-3-7091-1371-4

[67] Altenbach, H., Maugin, G. A., Erofeyev, V. (eds.) "Mechanics of Generalized Continua", 1st ed., Springer, Berlin, Germany, 2011. https://doi.org/10.1007/978-3-642-19219-7

[68] Eremeyev, V. A., Lebedev, L. P., Altenbach, H. "Foundations of Micropolar Mechanics", 1st ed., Springer, Berlin, Germany, 2013. https://doi.org/10.1007/978-3-642-28353-6

[69] Maugin, G. A. "Non-Classical Continuum Mechanics: A Dictionary", 1st ed., Springer, Singapore, 2017. https://doi.org/10.1007/978-981-10-2434-4

[70] Maugin, G. A., Metrikine, A. V. (eds.) "Mechanics of Generalized Continua. One Hundred Years after the Cosserats", 1st ed., Springer, New York, NY, USA, 2010. https://doi.org/10.1007/978-1-4419-5695-8

[71] Lámer, G. "Contradictions in the theory of micropolar elasticity and their causes", Newsletter, Technical University of Budapest, 2(1), pp. 12-16, 1984.

[72] Lámer, G. "Continuous and discrete models in the mechanics of deformable solid bodies", MATEC Web of Conferences, 184, Article ID: 01004,2018 https://doi.org/10.1051/matecconf/201818401004

[73] Capriz, G. "Continua with Microstructure", 1st ed., Springer, New York, NY, USA, 1989.

https://doi.org/10.1007/978-1-4612-3584-2

[74] Kantorovich, L. V., Krilov, V. I. "Approksimatsionnyye Metody Vysshego Analiza" (Approximation Methods of Higher Analysis), 3rd ed., GITTL, Moscow, Russia, 1950. (in Russian)

[75] Truesdell, C. "The Kinematics of Vorticity", Indiana University Press, Bloomington, Indiana, 1954.
[76] Frisch, U. "Turbulence", 1st ed., Cambridge University Press, Cambridge, UK, 1995. https://doi.org/10.1017/CBO9781139170666

[77] Lumley, J. L., Berkooz, G., Elezgaray, J., Holmes, P., Poje, A., Volte, C. "Fundamental aspects of incompressible and compressible turbulent flow", In: Gatski, T. B., Hussaini, M. Y., Lumley, J. L. (eds.) Simulation and Modeling of Turbulent Flows, Oxford University Press, New York, NY, USA, 1996, pp. 5-78.

[78] Leonard, A. "Direct numerical simulation of turbulent flows", In: Gatski, T. B., Hussaini, M. Y., Lumley, J. L. (eds.) Simulation and Modeling of Turbulent Flows, Oxford University Press, New York, NY, USA, 1996, pp. 79-108.

[79] Ferzinger, J. H. "Large eddy simulation", In: Gatski, T. B., Hussaini, M. Y., Lumley, J. L. (eds.) Simulation and Modeling of Turbulent Flows, Oxford University Press, New York, NY, USA, 1996, pp. 109-154.

[80] Smagorinsky, J. "General circulation experiments with the primitive equations: I. The basic experiment", Monthly Weather Review, 91(3), pp. 99-152, 1963. https://doi.org/10.1175/1520-0493(1963)091<0099:GCEWTP>2.3.CO;2

[81] Launder, B. E. "An introduction to single-point closure methodology", In: Gatski, T. B., Hussaini, M. Y., Lumley, J. L. (eds.) Simulation and Modeling of Turbulent Flows, Oxford University Press, New York, NY, USA, 1996, pp. 243-310.

[82] Andersson, B., Andersson, R., Håkansson, L., Mortensen, M., Sudiyo, R., van Wachem, B., Hellstrom, L. "Computational Fluid Dynamics for Engineers", 1st ed., Cambridge University Press, Cambridge, UK, 2012.

[83] Speziale, C. G. "Modelling of turbulent transport equations", In: Gatski, T. B., Hussaini, M. Y., Lumley, J. L. (eds.) Simulation and Modeling of Turbulent Flows, Oxford University Press, New York, NY, USA, 1996, pp. 185-242.

[84] Gatski, T. B., Speziale, C. G. "On explicit algebraic stress models for complex turbulent flows", Journal of Fluid Mechanics, 254, pp. 59-78, 1993. https://doi.org/10.1017/S0022112093002034

[85] Speziale, C. G. "On nonlinear $K-l$ and $K-\varepsilon$ models of turbulence", Journal of Fluid Mechanics, 178, pp. 459-475, 1987. https://doi.org/10.1017/S0022112087001319

[86] Lámer, G. "Notes on the theory of large displacement with small strain", Periodica Politechnica Civil Engineering, 29(1-2), pp. 53-65, 1985. 